\title{
Evoked potentials in cat auditory nerve: Suppression by prior tonal stimulation
}

\author{
JAY W. BAUER and ROBERT GALAMBOS \\ Departments of Psychology and Neuroscience \\ University of California, San Diego, La Jolla, California 92037
}

\begin{abstract}
Action potentials (AP) recorded from the eighth nerve of a cat to a tone pip, $\mathrm{S}_{1}$, were suppressed when preceded by a monotic or dichotic conditioning tone, Tc. The suppression increased when (1) the interval between Tc and $S_{1}$ was decreased, (2) either the intensity or the duration of Tc was increased, or (3) the difference in frequency between the two stimuli was reduced. Thus the nerve action potential amplitude correlates strongly, but inversely, with the loudness enhancement effects seen in man (Galambos, Bauer, Picton, Squires \& Squires, Journal of the Acoustical Society of America, 1972, 52). Since Coats and Dickey (Journal of the Acoustical Society of America, 1972, 52) found that the human AP can be diminished by a preceding noise without reducing the loudness of a click, the available results suggest that a central mechanism functions to compensate for the reduction in AP amplitude. The dichotic effects could be accounted for by cross-hearing, thereby providing no evidence for a role of the olivocochlear bundle in the effects observed.
\end{abstract}

When human subjects hear two brief auditory stimuli that follow one another in time, the first can enhance the loudness of the second (Buytendijk \& Meesters, 1942; Elmasian \& Galambos, 1973; Galambos, Bauer, Picton, Squires, \& Squires, 1972a, b; Gol'dburt, 1963; Irwin \& Zwislocki, 1971; Sokolich \& Zwislocki, 1972). Throughout this report, the first signal (a "conditioning tone") is designated Tc, the second signal (the "enhanced" one) is $S_{1}$, and the interval between them is $\Delta T$.

Galambos et al. (1972a, b) investigated several parameters in a dichotic loudness enhancement task by matching the loudness of $S_{1}$ with a third stimulus, $S_{2}$, which followed $S_{1}$ by $1,500 \mathrm{msec} . S_{1}(8 \mathrm{KHz}$, $2 \mathrm{msec}$ ) was enhanced by as much as $15 \mathrm{~dB}$ in these experiments. They found that loudness enhancement (1) disappeared as the duration of $\Delta \mathrm{T}$ was increased, (2) increased as the intensity or duration of Tc was increased, and (3) was greatest when the frequency of Tc was the same or nearly the same as the frequency of $S_{1}$. In the monotic loudness enhancement task, even greater enhancements of $20 \mathrm{~dB}$ or more have been obtained by Elmasian and Galambos (1973).

The following experiments examine the effect of Tc on the eighth nerve response to $S_{1}$ in a cat. They were done with stimulus parameters similar to the ones used in the loudness enhancement study of Galambos et al. (1972a, b), with the objective of determining the role of the auditory nerve in loudness enhancement. The $N_{1}-P_{1}$ component of the eighth nerve action potential (AP) was measured to $S_{1}$ in three conditions:

This research was supported by a grant from NIH (NS 10482-03). We thank Donald Norman for the use of his facilities. This paper is based on part of a thesis submitted by the first author to the University of California, San Diego, in partial fulfillment of the requirements for a $\mathrm{PhD}$ degree.
$S_{1}$ alone, $S_{1}$ with a monotic Tc, and $S_{1}$ with a dichotic Tc, while the different parameters of the stimulus configuration were varied. If loudness enhancement is mediated at the eighth nerve level, the variations in the amplitude of the AP to $S_{1}$ produced by Tc should correlate positively with the enhancements of $S_{1}$ loudness produced by $\mathrm{Tc}$ in man. As we shall see, they do not.

\section{METHODS AND PROCEDURES}

One healthy young female cat was used in this experiment. She was studied in about 402 - to 3-h sessions spread over a 3-month period. A bipolar electrode chronically implanted in her left-eighth nerve yielded all the data reported here. The electrode was a Teflon-coated stainless steel wire with a tip diameter of approximately $200 \mu$; it had been implanted 7 months earlier using a stereotaxic instrument and standard operating procedures. The bibolar pair and a grounding electrode (skull screw) were terminated in a subminiature connector attached to the skull. At a second operation, the middle ear muscles on the implanted side were sectioned by electrocautery without damage to the ossicles. Data recording began 2 months later.

Recordings were made with the cat unanesthetized and lightly restrained in a box inside a double-walled acoustic chamber. The bipolar recordings were first differentially amplified 100 times by a Grass P-15 with a bandpass of 30 to $3,000 \mathrm{~Hz}$ and were further amplified 100 times by a Tektronix 122 between $8 \mathrm{~Hz}$ and $10 \mathrm{KHz}$, for a total amplification factor of 10,000 . The output of the Tektronix was sampled by the analog-to-digital converter on a PDP. 9 computer to a 12-bit accuracy at a sample rate of $20 \mathrm{KHz}$. The output of a MB Type 302 accelerometer attached to the pedestal on the cat's head was amplified 100 times by a Grass P-15 between $.1 \mathrm{~Hz}$ and $1 \mathrm{KHz}$ and then sampled for $25 \mathrm{msec}$ at a $10-\mathrm{KHz}$ sampling rate by the A-to-D converter prior to the presentation of stimuli on each trial. If the maximum peak-to-peak voltage from the accelerometer was greater than $100 \mathrm{mV}$, which indicated that the cat was moving or making noises, the trial was not recorded.

Trials were presented in blocks of 40 with an intertrial time of $2 \mathrm{sec}$. The trials within a block alternated between trials with $S_{1}$ alone and trials with TC preceding $S_{1}$ by the interval $\Delta T$. On the 
monotic trials, $S_{1}$ and $T c$ were presented to the left ear, and on dichotic trials, $S_{1}$ was presented to the left and $T c$ to the right ear. Te was generated by a Wavetek 116 voltage control generator (VCG) and $S_{1}$ by a Wavetek 150 VCG. Both Tc and $S_{1}$ were band pass liltered by a Krohn-Hite $3550 \mathrm{R}$ set at $100 \mathrm{~Hz}$ above and below the stimulus frequency. The stimuli were delivered through audiovox $9 \mathrm{C}$ earphones positioned inside the pinna at the entrance to the external auditory meatus. Four different parameters were varied; $\Delta \mathrm{T}$ interval duration, $\mathrm{Tc}$ intensity, $\mathrm{Tc}$ duration, and $\mathrm{Tc}$ frequency (at two different $S_{1}$ frequencies). Intensity functions were obtained for $S_{1}$ presented to both the ipsilateral (left) and contralateral (right) ear in order to obtain a measure of the cross-hearing. The order of the parametric values presented and whether the condition was monotic or dichotic on a given block of trials was determined randomly. The usual values of all parameters except the one being investigated were as follows: $S_{1}, 80 \mathrm{~dB}$ SPL, $1 \mathrm{msec}$, and $8 \mathrm{KHz} ; \mathrm{Tc}, 100 \mathrm{~dB} \mathrm{SPL}, 250 \mathrm{msec}$, and $8 \mathrm{KHz}$; and $\Delta \mathrm{T}$ was $10 \mathrm{msec}$. All measures of intensity are for a continuous tone. $\Delta \mathrm{T}$ varied in the interval duration experiment between 10 and $500 \mathrm{msec}$. Tc in the intensity study ranged from 50 to $100 \mathrm{~dB}$ SPL in 10-dB steps and in the duration experiment from 1 to $500 \mathrm{msec}$. In the Tc frequency study, when $S_{1}$ was $8 \mathrm{KHz}$ Tc varied between 5 and $11 \mathrm{KHz}$ in $500-\mathrm{Hz}$ steps. In the second half of the experiment, $\mathrm{S}_{1}$ frequency was set at $5 \mathrm{KHz}$ and Tc frequency varied between 2 and $8 \mathrm{KHz}$ in $500-\mathrm{Hz}$ steps with no change in the other parameters. In the $S_{1}$ intensity series, the $S_{1}$ level ranged from 50 to $100 \mathrm{~dB}$ SPL in 10-dB steps. All stimulus presentations were controlled by the PDP-9 computer.

The eighth nerve action potentials (AP) recorded to $S_{1}$ by the computer's analog-to-digital converter were segregated depending upon whether or not Tc had been presented and then summed with like trials. On each trial, the computer measured and recorded the latency and peak-to-peak amplitude of each wave of the waveform. At the end of the block of trials, the computer calculated the means and standard deviations for the latencies and amplitudes for both the trials with and without Tc, and the difference between the trials with and without Tc for all the measurements. The difference in $\mathrm{N}_{1}-\mathrm{P}_{1}$ amplitude between $\mathrm{Tc}$ and no-Tc trials is the statistic reported here to demonstrate the effect of Tc on the eighth nerve response to $S_{1}$.

\section{RESULTS}

Figure 1 shows sample waveforms of the AP as a function of $S_{1}$ intensity when presented both ipsilaterally and contralaterally to the ear with the electrode. Note the similar morphology of the responses to ipsilateral and contralateral stimuli, and that the response to an ipsilateral stimulus is greater than that to a contralateral one. The fact that it takes an increase of $21 \pm 3 \mathrm{~dB}$ in the intensity of the contralateral $S_{1}$ to produce an $N_{1}-P_{1}$ amplitude equal to that of an ipsilateral $S_{1}$ demonstrates the amount of cross-hearing in this cat for $8-\mathrm{KHz} 1-\mathrm{msec}$ signals. Figure 2 plots the amplitude of the $N_{1}-P_{1}$ wave as a function of $S_{1}$ intensity for both conditions. The contralateral $S_{1}$ starts to generate a substantial response when its level is some $20 \mathrm{~dB}$ above that which produces a response in the ipsilateral case. Since both functions increase linearly and essentially in parallel thereafter, the cross-hearing effect of approximately $20 \mathrm{~dB}$ appears to be the same at 60 , 70,80 , and $90 \mathrm{~dB}$ as well.

The graph in Figure 3 plots the difference in $N_{1}-P_{1}$ amplitudes between $T c$ and no-Tc trials. A positive

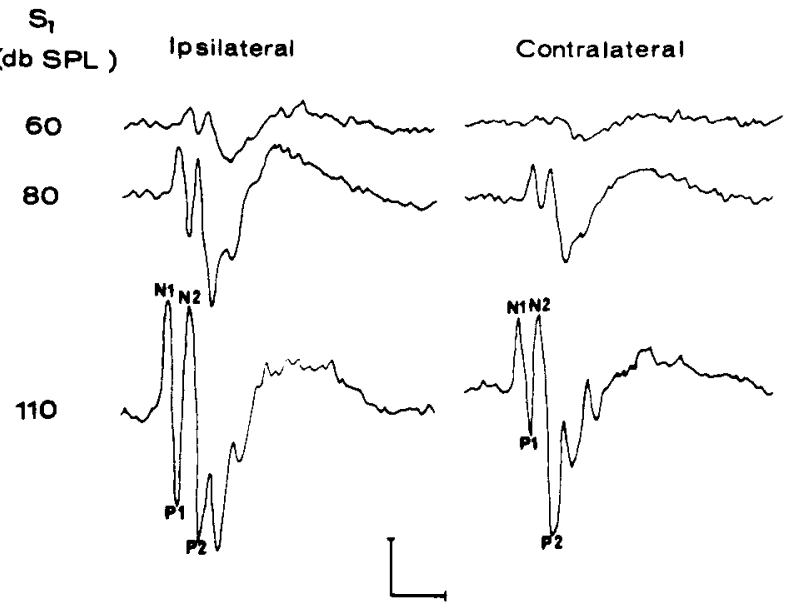

Figure 1. Cat eighth nerve action potentials to $S_{1}(1 \mathrm{msec}$, $8 \mathrm{KHz}$ ) presented monaurally to the ear ipsilateral and contralateral to the electrode site. Calibration: $100 \mu \mathrm{V}, 2$ msec.

value means that Tc enhanced the AP to $S_{1}$, while a minus value means that $\mathrm{Tc}$ suppressed it. Figure 3 shows that $\mathrm{Tc}$ reduces the amplitude of the $\mathrm{N}_{1}-\mathrm{P}_{1}$ wave in both the monotic and dichotic conditions. The ipsilateral $\mathrm{Tc}$ is more effective than the contralateral by $17 \pm 4 \mathrm{~dB}$ (as measured from the 70-, 80-, 90-, and $100-\mathrm{dB}$ points on the dichotic amplitude difference curve to the equal amplitude levels on the monotic curve). Since a $t$ test between the difference in cross-hearing seen in Figure 2 and the difference

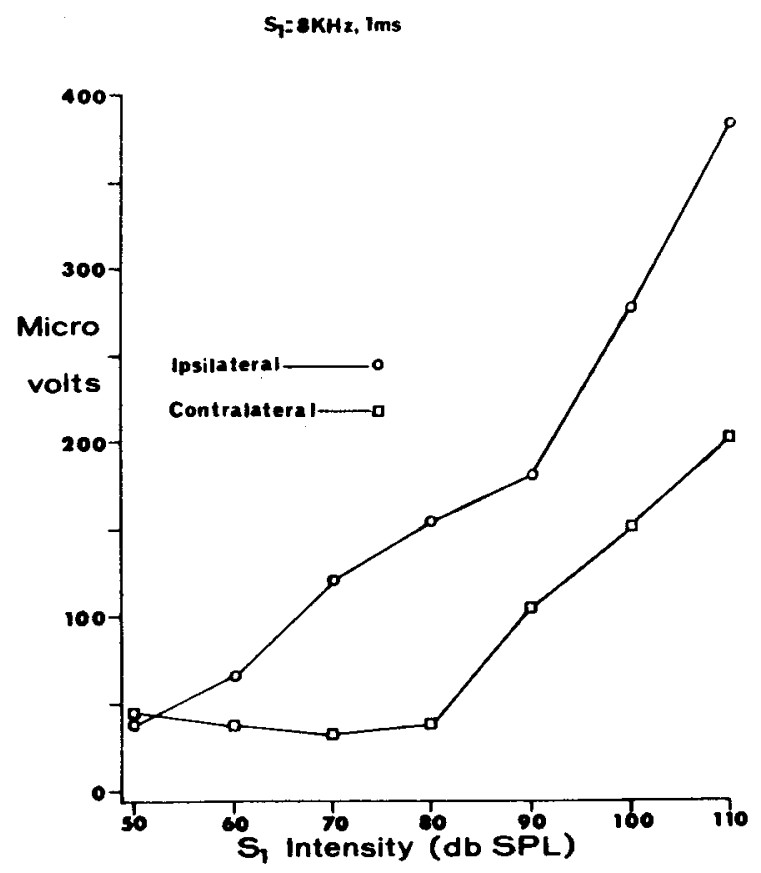

Figure 2. Amplitude of the $N_{1}-P_{1}$ component of the AP to the same signal $\left(S_{1}\right)$ presented ipsilaterally and contralaterally. 


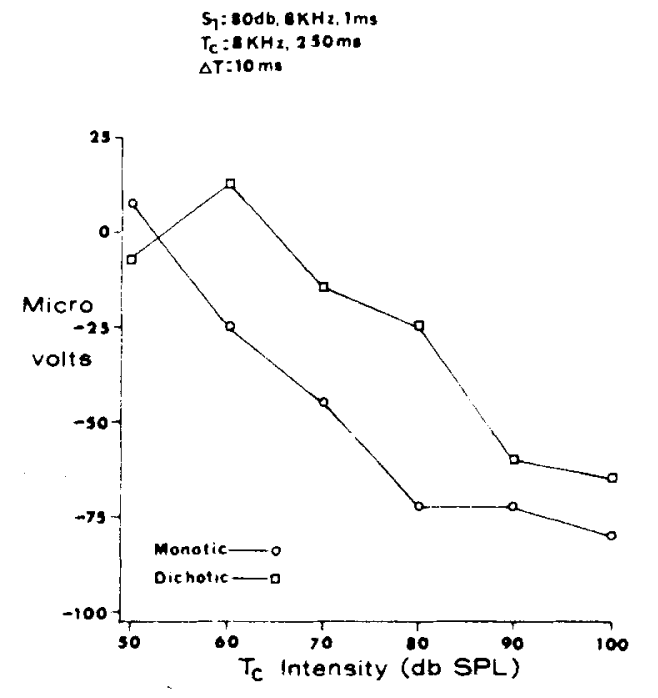

Figure 3. The reduction in the amplitude of the $N_{1}-P_{1}$ component to a signal $\left(S_{1}\right)$ as a function of the intensity of a prior stimulus (Te). Both the monotic and dichotic conditions are shown.

between the dichotic and monotic conditions of Figure 3 was insignificant, the effect of a contralateral Tc can be accounted for by cross-hearing alone.

The reduction in $N_{1}-P_{1}$ amplitude induced by $T c$ becomes smaller as $\Delta \mathrm{T}$ is increased, as shown in Figure 4 . When $\Delta \mathrm{T}$ reaches about 350 to $500 \mathrm{msec}$, the effect of $T c$ upon the response to $S_{1}$ disappears. Further examination of the curves shows that for short $\Delta \mathrm{T}$ intervals, $25 \mathrm{msec}$ or less, a monotic Tc suppresses $\mathrm{N}_{1}-\mathrm{P}_{1}$ amplitude by 40 to $60 \mu \mathrm{V}$ more than a dichotic $T c$, and that for longer $\Delta T$ intervals, the monotic and dichotic Tes produce about the same amount of reduction in $N_{1}-P_{1}$ amplitude. This suggests that there may be more than just cross-hearing involved in the dichotic condition.

Figure 5 shows that increasing $\mathrm{Tc}$ duration gradually reduces the amplitude of the AP to $S_{1}$ similarly in both the dichotic and monotic conditions. Each asymptotes at about $100 \mathrm{msec}$, beyond which there is no reduction in the AP. The average difference between the monotic and dichotic reductions in AP amplitude is $21 \pm 17 \mu \mathrm{V}$. The difference between the monotic and dichotic curves for the Tc intensity function is $27 \pm 14 \mu \mathrm{V}$. Since the dichotic effect in the Tc intensity function can be accounted for by cross-hearing, and since the difference in $\mathrm{Tc}$ effectiveness between the monotic and dichotic conditions for both the Te intensity and duration functions are statistically the same, there is no evidence that the dichotic effect in the Tc duration experiment is due to anything more than cross-hearing.

Varying the frequency of $T c$ away from the frequency of $S_{1}$ in either direction reduces its suppressive effect on $S_{1} \mathrm{~s}$ of either $8 \mathrm{KHz}$ (Figure 6) or $5 \mathrm{KHz}$ (Figure 7). When using a 5-KHz $S_{1}$, the $\mathrm{N}_{1}-\mathrm{P}_{1}$ amplitude is quite small, especially when $S_{1}$ was preceded by $\mathrm{Tc}$ (Figure 8). Therefore, $\mathrm{N}_{2}-\mathrm{P}_{2}$ was measured and the effect of varying Tc frequency on this component is plotted in Figure 7. At both 8 and $5 \mathrm{KHz}$, the maximum suppression occurs at the frequency of $S_{1}$ or just below it, and decreases as Tc frequency is varied away from $S_{1}$ frequency. All of the curves show a significantly steeper rise in the recovery from suppression for the frequencies above $S_{1}$ than for the frequencies below $S_{1}[T(10)=2.89, \mathrm{p}<.01$, monotic and $8 \mathrm{KHz} ; \mathrm{T}(10)=2.64, \mathrm{p}<.025$, dichotic and $8 \mathrm{KHz} ; \mathrm{T}(10)=4.63, \mathrm{p}<.005$, monotic and $5 \mathrm{KHz}$; and $\mathrm{T}(10)=2.85, \mathrm{p}<.01$, dichotic and $5 \mathrm{KHz}$. That the suppressive effects of Tc on the $S_{1}$ $\mathrm{AP}$ is frequency-dependent for two different frequencies of $S_{1}$ tends to confirm that the middle ear muscles were successfully sectioned, or at least not producing the effects observed in these experiments.

\section{DISCUSSION}

The objective of the experiments just reported was to determine whether the aldditory nerve plays a role in

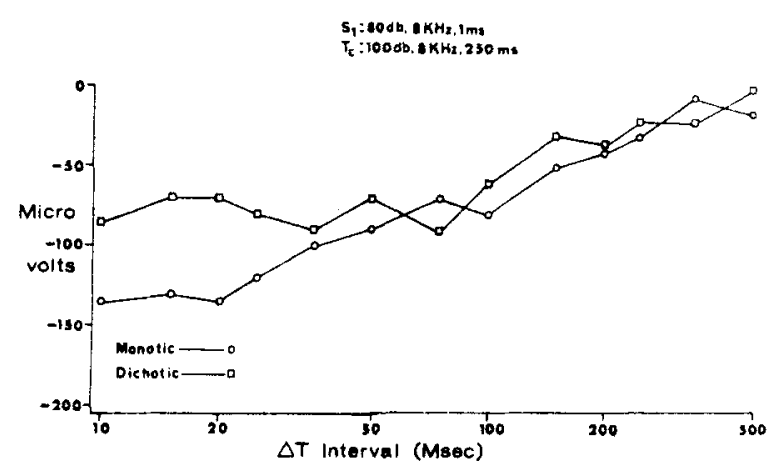

Figure 4. The reduction in amplitude of the $N_{1} \cdot P_{1}$ component to $S_{1}$ by a prior $T c$ as a function of $\Delta T$, the interval in milliseconds between $T c$ and $S_{1}$. Both the monotic and dichotic conditions are shown.

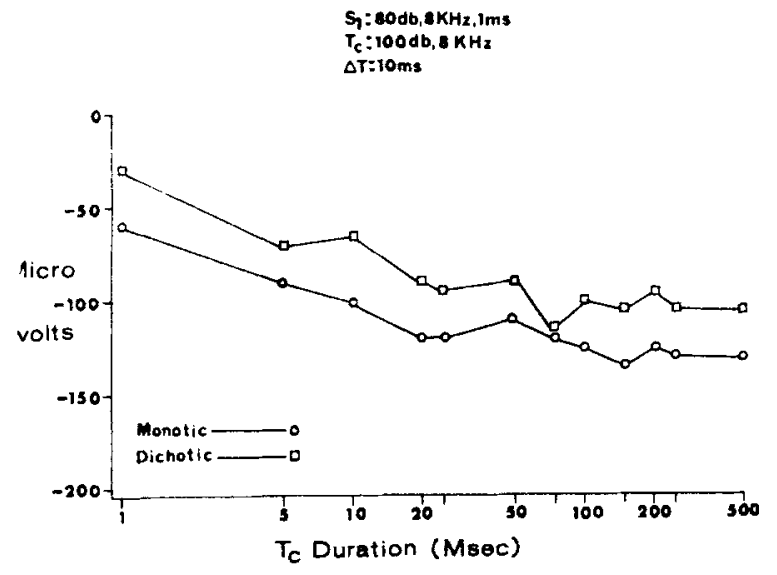

Figure 5. The reduction in amplitude of the $N_{1}-P_{1}$ component to $S_{1}$ as a function of the duration of a prior Tc. Both the monotic and dichotic conditions are shown. 


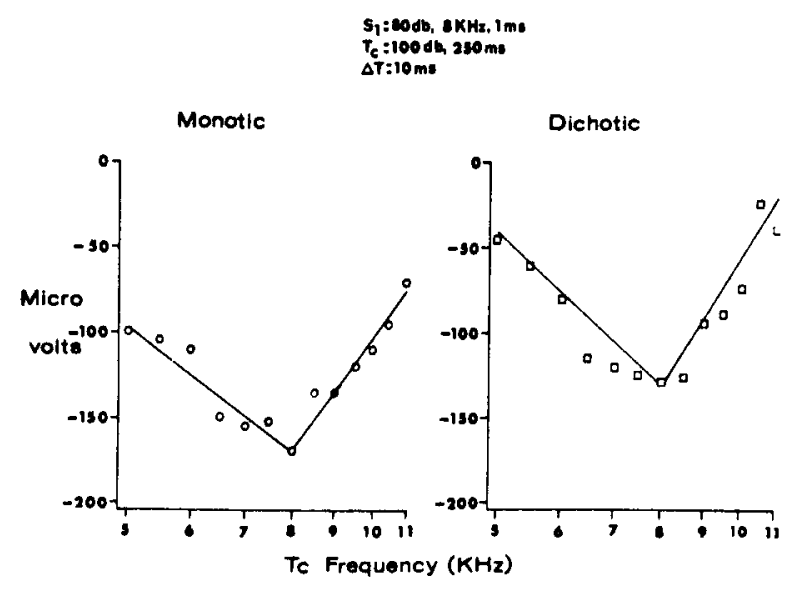

Figure 6. The reduction in the amplitude of the $N_{1}-P_{1}$ component to an $8-\mathrm{KHz} S_{1}$ as a function of the frequency of the prior stimulus, Tc. Both the monotic and dichotic conditions are shown.

\section{$S_{1}: 100 \mathrm{db}, 5 K \mathrm{KH}_{1}, \mathrm{~mm}$ $T_{C}: 100016,230 \mathrm{~ms}$} $\Delta T: 10 \mathrm{~ms}$

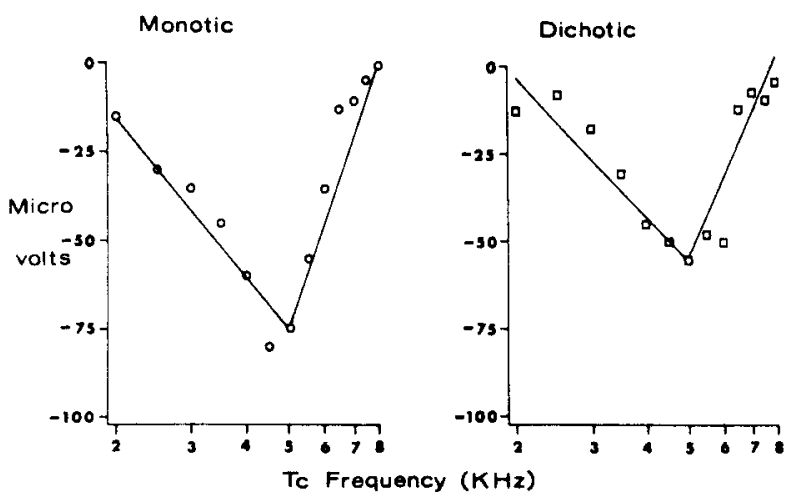

Figure 7. The reduction in amplitude of the $N_{2}-P_{2}$ component to a 5-KHz $S_{1}$ as a function of the frequency of the prior stimulus, Tc. Both the monotic and dichotic conditions are shown.

loudness enhancement. To do this, the AP generated by $S_{1}$ was examined in both the absence and presence of $\mathrm{Tc}$. The results suggest a strong, but inverse, correlation between the AP amplitude and loudness enhancement. For every parametric variation that increases the enhancement effect in man (increasing $T c$ intensity or duration, shortening the $\Delta T$ interval, or matching the frequency of Tc to that of $S_{1}$ ) there is a greater suppression of the $S_{1}$ AP amplitude in cat.

That Tc consistently suppresses the AP to $S_{1}$ suggests that the cat perceived $S_{1}$ to be masked by Tc. In man, however, a study on the recovery of both click loudness and AP amplitude by Coats and Dickey (1972) suggests that this may not be the case. They found that, although the amplitude of the human AP to a click is reduced by a preceding burst of noise and has not completely recovered after a $\Delta T$ interval of $500 \mathrm{msec}$, its loudness recovered within $150 \mathrm{msec}$ of the offset of Tc. ${ }^{1}$ This shows that the AP amplitude cannot be equated with the perceived loudness of a stimulus and so it is not impossible that the cat in our study actually perceived $S_{1}$ as enhanced. Coats and Dickey (1972) suggested a way to reconcile the differences between the recovery functions of the AP and the loudness of the click. They proposed that there was a more central mechanism which compensates for the reduction $\mathrm{Tc}$ produces in the AP to $S_{1}$ so that the "suppressed" $S_{1}$ is perceived to be as loud as a control $S_{1}$ without Tc. If such a mechanism were to overcompensate for the suppressive effects of $T c$ on $S_{1}$, then loudness enhancement could occur despite the results obtained here.

The relationship between the present electrophysiological results and loudness enhancement contrasts strongly with the relationship between eighth nerve AP amplitude and auditory sensitization (Hughes, 1954; Hughes \& Rosenblith, 1957). Auditory sensitization (Hughes, 1954) is a lowering of the psychophysical threshold of tone pips produced by a preceding intense tone of several minutes' duration. Hughes and Rosenblith (1957) have shown a strong, positive correlation between the variations in AP amplitude produced by a preceding "sensitizing" tone in cats and the time course of the auditory sensitization effect observed in man under a variety of similar stimulus conditions. This suggests that these two similar psychophysical phenomena-sensitization and enhancement - are quite different in that one is probably due to a peripheral mechanism while the other seems to be due to a more central process.

Since the effects of both an ipsilateral and contralateral $\mathrm{Tc}$ on the AP amplitude of $S_{1}$ were investigated, it is possible to assess the role, if any, of the olivocochlear bundle (OCB) on the effects observed here. Galambos (1956) and Desmedt (1962) have demonstrated that shocking the crossed OCB reduces the AP of the eighth nerve to clicks. And

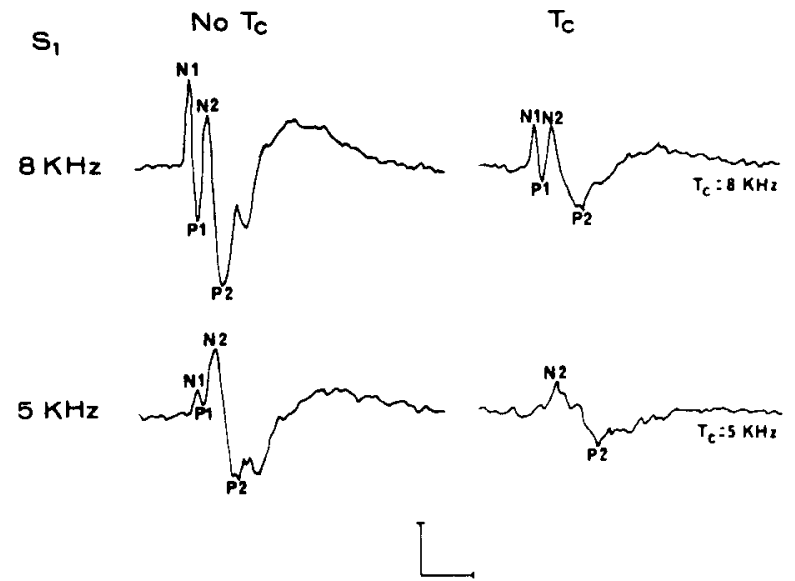

Figure 8. Eighth nerve action potentials to an 8- and a 5-KHz $S_{1}$ $(1 \mathrm{msec}, 80 \mathrm{~dB})$ with and without a monotic Tc $(250 \mathrm{msec}, 100 \mathrm{~dB})$ of 8 and $5 \mathrm{KHz}$, respectively. Calibration: $100 \mu \mathrm{V}, 2$ msec. 
Neider and Neider (1970) showed that APs to clicks reduced by presenting noise return to their full amplitude when the OCB is shocked. It has also been demonstrated that sounds to one ear can activate both the crossed (Fex, 1962) and uncrossed (Fex, 1965) $O C B$. It seems, however, unlikely that the effects observed here can be attributed to the action of the OCB. Our findings on the effects of Tc (its intensity and duration, whether delivered to the ipsilateral or contralateral ear) are all statistically consistent with the idea that they are due solely to cross-hearing. To be sure, in the $\Delta T$ experiment, the monotic and dichotic curves converge at $\Delta T$ s longer than 25 or $30 \mathrm{msec}$, which suggests that something in addition to cross-hearing might be occurring in the dichotic condition. However, an explanation simpler than one invoking the $\mathrm{OCB}$ is available. Rosenzweig and Rosenblith (1953) demonstrated that the recovery functions to monotic Tes of different intensities converge as $\Delta \mathrm{T}$ is increased. If the contralateral $\mathrm{Tc}$ in the present studies is considered to be just a weaker, ipsilateral $\mathrm{Tc}$, then the convergence of the monotic and dichotic curves is also consistent with the cross-hearing hypothesis. Consequently, there is no evidence from these studies that the OCB plays a role in the suppressive effects of $T c$ observed here.

An interesting aspect of the suppressive effect of Tc on the AP to $S_{1}$ is its frequency specificity, which earlier investigators have not reported. Coats (1964) found no "qualitative" effects of varying Tc frequency on the suppression of the AP produced by a .01-msec square-wave $S_{1}$. Rosenblith, Galambos, and Hirsh (1950) found no systematic differences between Tc frequencies of $200,500,1,000$, and $4,000 \mathrm{~Hz}$ on the recovery of $\mathrm{AP}$ amplitude to a $.1-\mathrm{msec}$ pulse $S_{1}$. In both of these prior studies, the $S_{1}$ being suppressed had a broad energy spectrum, so it would not be expected that tonal stimuli would show differential suppressive effects; by contrast, the 1-msec tone pips used in this study have approximately $90 \%$ of their energy concentrated between $1,000 \mathrm{~Hz}$ above and below the nominal frequency of $S_{1}$ (Wightman, 1971). As we show, recovery from the suppression produced by $T c$ is more rapid when $T c$ is higher than $S_{1}$ in frequency, which agrees with the well-known observation that low frequencies mask high frequencies better than highs mask low (Egan \& Hake, 1950; Wegel \& Lane, 1924).

\section{REFERENCES}

Buytendik, F., \& Meester, A. Duration and course of the auditory sensation. Commetationes Pontifica Academic Scientiarum, 1942, 6, 557-576.

CoAts, A. Physiological observations of auditory masking. 1. Fifect of masking duration. Jound of Newrophysiology. 1964. 27. $986-1000)$.

CoATs, A., \& Dickey, J. Postmasking recovery of human click action potentials and click loudness. Joumal of the Acoustical Society of America, 1972, 52, 1607-1612.

Desmedt, J. Auditory-evoked potentials from cochlea to cortex as influenced by activation of the efferent olivocochleat bundle. Journal of the Acoustical Society of America, 1962, 34, 1478-1496.

EGAN, J., \& HaKe, H. On the masking pattern of a simple auditory stimulus. Journal of the Acoustical Society of America, 1950, 22, 622-630.

Elmasian, R., \& Galambos, R. Loudness enhancement with monaural, binaural and dichotic stimulation. Journal of the Acoustical Society of America, 1973, 53, 335(A).

FEx, J. Auditory activity in centrifugal and centripetal cochlear fibers in cat: A study of a feedback system. I. Acta Physiologica Scandinavica, 1962, Supplement 189.

Fex, J. Auditory activity in uncrossed centrifugal cochlear fibers in cat: A study of a feedback system. II. Acta Physiologica Scandinavica, 1965, 64, 43-57.

Galambos, R. Suppression of auditory nerve activity by stimulation of efferent fibers to the cochlear. Joumal of Neurophysiology, 1956, 19, 424-437.

Galambos, R., Bauer, J., Picton, T., Souires, K., \& SQuires, N. Lcudness enhancement following contralateral stimulation. Joumal of the Acoustical Society of America, 1972, 51, 141(A). (a)

Galambos, R., Bauer, J., Picton, T., Squires, K., \& SQuires, N. Loudness enhancement following contralateral stimulation. Journal of the Acoustical Society of America, 1972, 52, 1127-1130. (b)

Gol'DBURT, S. Discrimination between the order and loudness of two short tones in relation to their duration and the interval between them. Federation Proceedings, 1964, 23, T1206-T1210.

Huguts. J. Auditory sensitisation. Journal of the Acoustical Socitity of America, 1954, 26. 1064-1070.

Hughes, J., \& Rosenblith, W. Electrophysiological evidence for auditory sensitization. Journal of the Acoustical Society of America, 1957, 29, 275-280.

IRWIN, R., \& ZwisLockI, J. Loudness effects in pairs of tone bursts. Perception \& Psychophysics, 1971, 10, 189-192.

Nieder, P., \& Nieder, I. Antimasking effect of crossed olivocochlear bundle stimulation with loud clicks in guinea pig. Experimental Neurology, 1970, 28, 179-188.

Rosenblith, W., Galambos, R., \& Hirsh, I. The effect of exposure to loud tones upon animal and human responses to acoustic clicks. Science, 1950,$111 ; 569-571$.

Rosenzweig, M., \& Rosenblith, W. Responses to successive auditory stimuli at the cochlea and at the auditory cortex. Psychological Monographs, 1953, 67, 1-26.

Sokolich, W., \& ZwislockI, J. On loudness enhancement of a tone burst by a preceding tone burst. Joumal of the Acoustical Society of America, 1972, 52, 141-142(A).

WEgEL, R., \& LANE, C. The auditory masking of one pure tone by another and its probable relation to the dynamics of the inner ear. Physics Review, 1924, 23, 266-285.

WIGHTMAN, F. Detection of binaural tones as a function of masker bandwidth. Journal of the Acoustical Society of America, 1971, 50, 623-636.

\section{NOTE}

1. One might expect that the subjects of Coats and Dickey (1972) would have shown loudness enhancement, which they do not report. However, in their study, the differences in intensity between Tc and

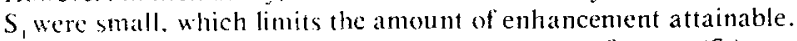
Funthemore, their interval between $S_{1}$ and the reference $\left\{S_{2}\right\}$ was often less than $1 \mathrm{sec}$, which means that $S_{2}$ itself could have been enhanced. Finally, it is at this time not known how well a noise $\mathrm{Tc}$ like theirs enhances a click $S_{1}$, nor how much is produced by the 3 -see $T_{c}$ they used. Consequently, their fallure to report enhancement may have been due to an unfortunate choice of stimulus parameters.

(Received for publication May 13, 1974; revision accepted August 25, 1974.) 